\title{
Radio emission from the bow shock of G2
}

\author{
P. Crumley ${ }^{1}$ and P. Kumar ${ }^{2}$ \\ ${ }^{1}$ Physics Department, University of Texas at Austin, Austin, TX 78712, USA \\ email: crumleyp@physics . utexas .edu \\ ${ }^{2}$ Astronomy Department, University of Texas at Austin, Austin, TX 78712, USA
}

\begin{abstract}
The radio flux from the synchrotron emission of electrons accelerated in the forward bow shock of G2 is expected to have peaked when the forward shock passes close to the pericenter from the Galactic center, around autumn of 2013. This radio flux is model dependent. We find that if G2 were to be a momentum-supported bow shock of a faint star with a strong wind, the radio synchrotron flux from the forward-shock heated ISM is well below the quiescent radio flux of Sgr A*. By contrast, if G2 is a diffuse cloud, the radio flux is predicted to be much larger than the quiescent radio flux and therefore should have already been detected or will be detected shortly. No such radiation has been observed to date. Radio measurements can reveal the nature of G2 well before G2 completes its periapsis passage.
\end{abstract}

Keywords. Galaxy: center — black hole physics — radiation mechanisms: nonthermal — shock waves

\section{Introduction}

G2, a spatially-extended red source, is on a nearly radial orbit headed towards the supermassive black hole at the Galactic center, Sgr A* (Gillessen et al. 2012, Gillessen et al. 2013, Phifer et al. 2013). As G2 plunges towards Sgr A*, it is supersonic with a Mach number of around 2. Therefore, G2 drives a bow shock into the hot ISM which is expected to accelerate electrons to relativistic energies. These high-energy electrons then produce synchrotron radiation in the radio band (Narayan et al. 2012, Sạdowski et al. 2013b). The synchrotron flux peaks when the forward shock reaches periapsis, where the magnetic field is strongest. The forward shock will reach periapsis $\sim 7$ months before the G2 center of mass does, so the forward shock emission should have peaked around autumn of 2013 (Sa̧dowski 2013a). The magnitude of the radio flux depends on how many electrons are swept up into the shock and therefore on the cylindrical size and nature of G2.

The nature of G2 is undetermined. When first discovered, Gillessen, et al. (2012) hypothesized that G2 was a pressure-confined, non-self-gravitating gas cloud, due to the fact that the Brackett-gamma $(\mathrm{Br}-\gamma)$ luminosity of $\mathrm{G} 2$ is not changing with time, $L_{\mathrm{Br}-\gamma} \sim 2 \times 10^{-3} \mathrm{~L}_{\odot}$, and the $\mathrm{Br}-\gamma$ velocity dispersion is increasing in a manner that is well fit by a gas cloud with a radius of $\sim 2 \times 10^{15} \mathrm{~cm}$ being tidally sheared by Sgr A*. Alternatively, there is another class of models where G2 contains a very faint stellar core that emits gas as it falls towards Sgr A* (Murray-Clay \& Loeb 2012, Scoville \& Burkert 2013, Ballone et al. 2013). The ionized gas is then tidally sheared and is the source of the Br- $\gamma$ radiation seen as G2. According to Scoville \& Burkert (2013), the ionized gas that is the source of the $\mathrm{Br}-\gamma$ radiation is located in the cold dense inner shock of a momentum-supported bow shock between a stellar wind from a hidden, TTauri star and the hot ISM. 

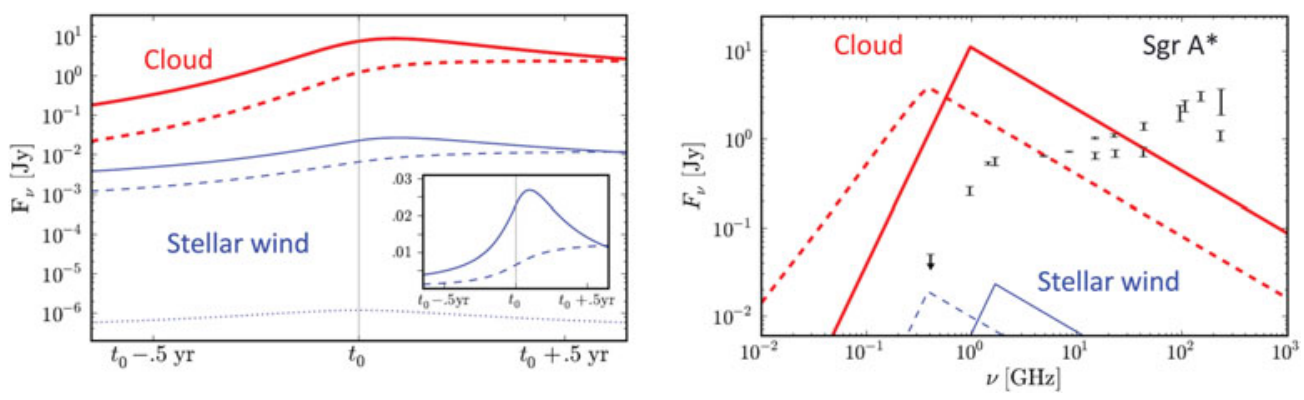

Figure 1. Left: The expected synchrotron flux at $1.4 \mathrm{GHz}$ around pericenter passage of the forward shock, $t_{0}$, for different models of G2. Solid lines are the predicted fluxes when all of the accelerated electrons stay inside the bow shock. Dashed lines are the predicted fluxes when the electrons quickly leave the bow shock region after being accelerated. Thick red lines are the predicted fluxes assuming that G2 is a diffuse cloud and correspond to the larger prediction of the radio flux by Sądowski, et al. (2013b). The lower blue lines are the predicted fluxes if G2 is a shocked stellar wind. The dotted blue line is the predicted flux from electrons accelerated in the inner, reverse shock. Left Inset: A linear scale plot showing the flux at $1.4 \mathrm{GHz}$ in Jy if G2 is a stellar wind. Right: Spectra at a time $t_{0}+0.05$ yr., where $t_{0}$ is the periapsis crossing time of the forward shock for different models of G2. The color scheme is the same as left except the flux from the inner shock is omitted. The data points are radio fluxes measured during periods of inactivity of Sgr A* (Davies et al. 1976, Falcke et al. 1998, Zhao et al. 2003). [A COLOR VERsion is aVAilable ONLINe.]

\section{Methodology}

To calculate the number of electrons swept into the bow shock, we assume that the ISM particle number density and temperature are inversely proportional to the distance from Sgr A*. We calculate the size of the bow shock if G2 is a shocked stellar wind by assuming it remains in pressure equilibrium with the ISM. We use Ballone et al. (2013) wind properties, with a mass-loss rate of $\dot{M}_{\mathrm{w}}=8.8 \times 10^{-8} \mathrm{M}_{\odot} / \mathrm{yr}$ and a velocity $v_{\mathrm{w}}=50 \mathrm{~km} \mathrm{~s}^{-1}$. In the cloud model we assume the area is equal to $\pi \times 10^{30} \mathrm{~cm}^{2}$, as suggested by Narayan, et al. (2012). If the cloud stays in pressure equilibrium, this area may be smaller which would reduce the synchrotron flux in the cloud model (Shcherbakov 2013). To calculate the synchrotron flux, we extend the methodology of Sadowski, et al. (2013b) to the case where the bow shock area is changing. Using Sądowski, et al. (2013b) particle-in-cell simulations, we assume that $5 \%$ of electrons are accelerated into a power law with a starting Lorentz factor $\gamma=7.5 k T / m_{e} c^{2}+1$, where $T$ is the unshocked ISM temperature. We assume $P_{\mathrm{mag}}=\chi P_{\mathrm{gas}}, \chi \approx 0.1$.

\section{Results}

We find that for both considered models of G2, the radio flux of forward shock of G2 peaks shortly after forward shock periapsis crossing (see Figure 1). If G2 is a shocked stellar wind, the radio flux will peak at a value around $0.02 \mathrm{Jy}$, which is far below the radio flux of Sgr A*. This flux scales linearly with the wind parameters $\dot{M}_{\mathrm{w}}$ and $v_{\mathrm{w}}$, as well as the fraction of electrons that are accelerated.

\section{References}

Ballone A., et al. 2013,ApJ 776, 13

Crumley P. \& Kumar P. 2013, MNRAS 436, 1955

Davies R. D., Walsh D., \& Booth R. S. 1976, MNRAS 177, 319 
Falcke H., et al. 1998, ApJ 499, 731

Gillessen S., et al. 2012, Nature 481, 51

Gillessen S., et al., 2013, ApJ 774, 44

Murray-Clay R. A. \& Loeb A. 2012, Nature Communications 3, 1049

Narayan R., Özel F., \& Sironi L. 2012, ApJ 757, L20

Phifer K., et al. 2013, ApJ 773, L13

Sądowski A., Narayan R., Sironi L., \& Özel F. 2013a, MNRAS 433, 2165

Sądowski A., Sironi L., Abarca D., Guo X., Özel F., \& Narayan R. 2013, MNRAS 432, 478

Scoville N. \& Burkert A. 2013, ApJ 768, 108

Shcherbakov, R. V. 2013, arXiv: 1309.2282

Zhao J. H., et al. 2003, ApJ 586, L29 